\title{
ISOLATION AND CHARACTERIZATION OF CANINE PARVOVIRUS TYPE 2C (CPV-2C) FROM SYMPTOMATIC PUPPIES
}

\author{
Puentes, $\mathbf{R}^{1 *}$; Eliopulos, $\mathbf{N}^{1}$; Pérez, $\mathbf{R}^{2}$; Franco, $\mathbf{G}^{1}$; Sosa, $\mathrm{K}^{2}$; Bianchi, $\mathbf{P}^{2}$; Furtado, $\mathbf{A}^{1}$; Hübner, S.O. ${ }^{3}$; Esteves, P.A. ${ }^{4}$ \\ ${ }^{1}$ Departamento de Ciencias Microbiológicas, Facultad de Veterinaria, UdelaR, Montevideo, Uruguay; ${ }^{2}$ Sección Genética \\ evolutiva, Facultad de Ciencias, UdelaR, Montevideo, Uruguay; ${ }^{3}$ Faculdade de Veterinaria, Universidade Federal de Pelotas, \\ Pelotas, RS, Brasil; ${ }^{4}$ Empresa Brasileira de Pesquisa Agropecuária, Concórdia, SC, Brasil.
}

Submitted: February 02, 2011; Returned to authors for corrections: April 14, 2011; Approved: June 07, 2012.

\begin{abstract}
Canine parvovirus type 2 (CPV-2) is a leading cause of diarrhea in puppies in several parts of the world. In this study CPV-2 was detected and recovered from puppies showing clinical disease from Montevideo, Uruguay. Samples were processed and used to infect CRFK and MDCK cells in order to isolate the virus. Out of twelve, two samples were positive for CPV-2. A genomic region of $583 \mathrm{bp}$ was amplified and the molecular characterization was performed by sequencing, phylogenetic analysis and Restriction Fragment Length Polymorphism (RFLP). Two isolated viruses (UY1 and UY2) were CPV-2c-like viruses. The comparison between the cytophatic effect (CPE) of CPV-2 (vaccinal virus) and CPV-2c (isolated virus) on primary canine cells cultures and on CRFK line cells, demonstrated that CPV-2c is less citopathogenic in CRFK than in primary cultures. Our study represents the first report on isolation and characterization of canine parvovirus type $2 \mathrm{c}(\mathrm{CPV}-2 \mathrm{c})$ in cell cultures from South American dogs.
\end{abstract}

Key words: Canine Parvovirus 2c, Isolation, Molecular characterization, South America.

\section{INTRODUCTION}

Parvovirus is regarded as one of the major causes of viral enteritis in puppies. The disease is caused by canine parvovirus type 2 (CPV-2). The virus infects the intestinal epithelium, producing necrosis of the crypts and atrophy of the villi. The affected animals show symptoms of depression, vomiting and diarrhea (mucoid to hemorrhagic gastroenteritis), which can lead to death within a few days. CPV-2 (Parvoviridae family) is a small virus with a single-stranded DNA genome of 5.2 kilobases $(\mathrm{kb})$ that encodes four peptides, two nonstructural (NS1, NS2) and two structural (VP1, VP2) (8). VP2 is the major component of the nonenveloped icosahedral capsid of CPV, and only a few substitutions in its amino acid (aa) sequence can alter relevant biological characteristics of the virus and generate new genotypes (CPV-2a, CPV-2b and CPV2c) $(1,2)$.

In 2001, a new genotype (CPV-2c) firstly described in Italy (4) has been recently detected in several countries, including Uruguay (8). This genotype can be differentiated

*Corresponding Author. Mailing address: Departamento de Ciencias Microbiológicas, Facultad de Veterinaria - UdelaR, Montevideo, Uruguay.; Tel.: +598-26281303; Fax: +598-2-6281303.; E-mail: rpuentes@adinet.com.uy 
from the others by Restriction Fragment Length Polymorphism (RFLP) analyses and sequencing (1). Although CPV-2 genotype seems to have disappeared from the nature, it is still used in most current commercial vaccines manufacture (11).

The aims of this study were the isolation and characterization of canine parvovirus circulating strains, from clinically ill dogs samples in Montevideo, Uruguay.

\section{MATERIALS AND METHODS}

\section{Samples and virus}

Twelve samples were collected by rectal swab from twelve dogs of various breeds and ages (up to 6 months) from Montevideo, Uruguay. All dogs had vaccination record (The pet owners said they had been fully vaccinated with CPV-2, although we have no certain about that) and showed hemorrhagic gastroenteritis, compatible with infection by canine Parvovirus. The samples were immersed in sterile phosphate-buffered saline (PBS) with antibiotic $(100 \mathrm{mg} / \mathrm{ml}$ penicillin and $100 \mathrm{U} / \mathrm{ml}$ streptomycin) and the suspension was centrifugated at $12,000 \mathrm{x}$. The cleared supernatant was filtered and frozen at $-80{ }^{\circ} \mathrm{C}$ until processing. As a positive control, the Cornell reference strain (ATCC - VR2017, CPV-2) was used.

\section{Virus isolation}

Crandall Ress feline kidney cells (CRFK, American Type Culture Collection, USA) was used in the virus isolation procedures. The samples were inoculated at a rate of $100 \mu \mathrm{L}$ per well in a cell's monolayer in maximum growth phase (12 hours of culture) with a low cell confluence (60\%). After adsorbed for 1 hour at $37^{\circ} \mathrm{C}$, the inoculum was removed, and minimum essential medium with Eagle salts was added (EMEM, Sigma-Aldrich ${ }^{\circledR}$, USA), then the cells were again incubated at $37^{\circ} \mathrm{C}$. Cell cultures were observed daily during 4 to 5 days to monitor the appearance of cytopathic effects. Subsequently the plate was frozen at $-80{ }^{\circ} \mathrm{C}$ and submitted to further passages following the same procedure, until the 5 th passage or the eventual appearance of cytopathic effect.

\section{Viral hemagglutination (HA)}

HA technique was performed as described by Senda et al. (9). Cornell strain (ATCC - VR2017) was used as a positive reference control. The viral titer was expressed as the highest dilution that caused complete hemagglutination.

\section{Polymerase Chain Reaction (PCR)}

PCR technique was performed as described by Buonavoglia et al. (1). The PCR amplified a VP2 gene fragment of 583 bp (position 4003 to 4585), using the primers: Forward 5' CAGGAAGATATCCAGAAGGA 3' (from 4003 to 4022) and Reverse 5' GGTGCTAGTTGATATGTAATAA ACA 3' (from 4585 to 4561 ).

\section{Restriction Fragment Length Polymorphism (RFLP) analysis}

For RFLP analysis, amplicons were digested with 5 units of the Mbo II restriction enzyme (Fermentas ${ }^{\circledR}$ ) according to the manufacturer's instructions. The cleavage pattern of the amplicons was observed on $1.5 \%$ agarose gels stained with ethidium bromide.

\section{Nucleotide and amino acid (aa) sequence analysis}

The sequence was performed as described by Perez et al. (8). Nucleotide and amino acid (aa) sequence alignment was performed by ClustalW method with BioEdit Sequence Alignment Editor (http://www.mbio.ncsu.edu/BioEdit/bioedit. html). For comparison purposes, the nucleotide sequences of CPV-2c (AY380577, AY742942 and BAD34656) and CPV-2a (DQ340434) isolates were retrieved from the GenBank.

\section{Citopathogenic viral effect on primary cell culture and CRFK}

In order to know the amount of citopathogenic effect 
produced by CPV-2c on primary cell cultures and line cells, we developed primary cell cultures from fetal canine heart $(\mathrm{FCH})$. The tissues were kindly donated to the University by veterinary doctors, obtained from routine surgical maneuvers performed on pregnant bitches (spaying). The CPE produced in these cell cultures was compared with the one that occurs in CRFK line cells, using the Cornell strain (CPV-2, ATCC - VR2017) as control.

Small fetal canine heart slices were cultured as explants with E-MEM supplemented with 20\% FBS. A fibroblasts monolayer was generated, then spread and used to infect with CPV-2 and CPV-2c in 24 wells plates $\left(\mathrm{Nunc}^{\circledR}\right)$. To assess the quantity of $\mathrm{CPE}$ produced by each virus, this scale was designed: + (low CPE), ++ (medium CPE), +++ (high CPE).

\section{RESULTS}

In two from twelve samples processed was possible to isolate canine parvovirus (UY1 and UY2) in CRFK cells. The presence of the virus in the cell cultures was confirmed by cytopathic effect (CPE), viral hemagglutination (HA) and PCR.

The HA showed a titer of 320 and 1280 in each sample. A single DNA band of the expected size (583 bp), corresponding to the partial amplification of the VP2 gene, was observed by gel electrophoresis in both samples. While CPV-2c genotypes have a Mbo II restriction site in the position 4062 to 4066 of the VP2, the restriction of such amplified fragment generates three fragments of $16 \mathrm{bp}, 55 \mathrm{bp}$ and $512 \mathrm{bp}$, the digestion of the amplicon in CPV-2/2a/2b produces only two fragments of 567 bp and 16 bp. The RFLP analysis showed that the two isolated CPV2 (UY1 and UY2) had a CPV-2c pattern (Fig. 2).

The comparative sequence analyses were performed using a 516-bp fragment (4023-4538) of the 583 amplicon that codes for 172 aa $(413-584)$ of the VP2 protein. The sequence alignment of the Uruguayan CPV-2c indicated that the strains UY1 and UY2 exhibited 100\% nucleotide identity with the CPV-2c from Italy and Germany. Both isolates (UY1 and UY2) revealed the presence of a GAA codon at position 426 of the VP2 protein. This GAA triplet codes for Glutamate, which indicates that these strains were type $2 \mathrm{c}$ as described previously (8).

Comparing the CPE produced by CPV-2c (UY1 and UY2 strains) with the CPE produced by $\mathrm{CPV}-2$, it was seen that $\mathrm{CPV}-2 \mathrm{c}$ is more cytopathogenic in fetal canine heart primary cell cultures $(\mathrm{FCH})$ than $\mathrm{CPV}-2$. Nevertheless the $\mathrm{CPE}$ produced by CPV-2 was similar in both, CRFK and FCH cells. It is also important to point that the isolated viruses, both UY1 and UY2, produce a low CPE in CRFK cells (Fig 1).

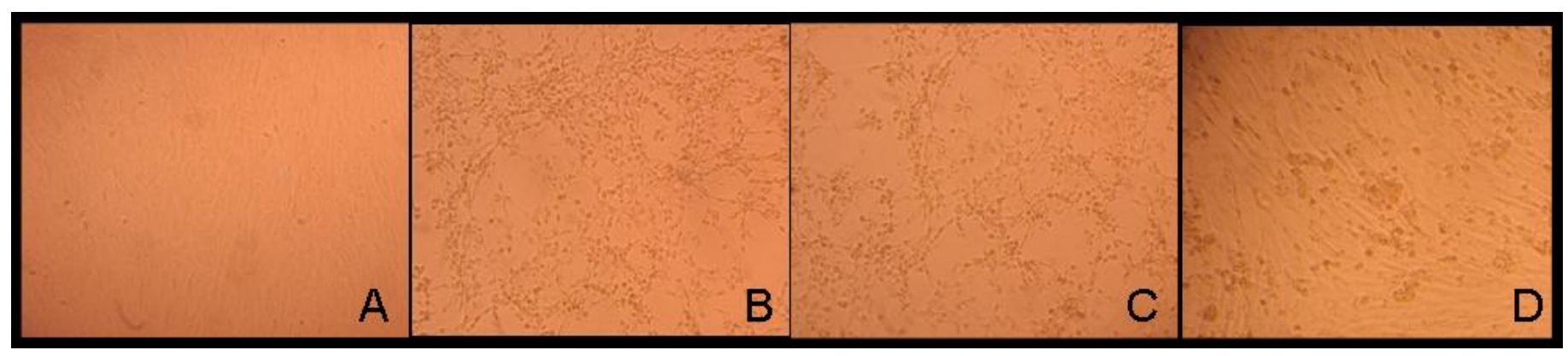

Figure 1. Citopathogenic effect of CPV-2c on cell culture. A) Uninfected cell control B) CRFK infected with CPV-2 (VR2017, reference strain) C) FCH infected with UY1 (CPV-2c) D) CRFK infected with UY1 (CPV-2c). 


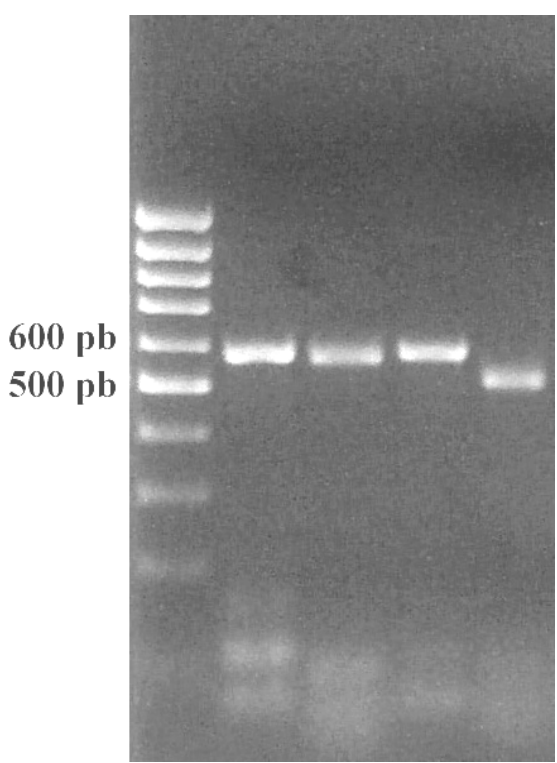

Figure 2. RFLP analysis of 555for/555rev amplicons. 1) 100 $\mathrm{kb}$ ladder, 2) undigested CPV-2 vaccine strain, 3) vaccine strain digested with $M b o$ II (CPV-2a and CPV-2b present the same pattern), 4) undigested culture isolate, 5) Culture isolate digested with $M b o I I$.

\section{DISCUSSION}

Nowadays there is a particular interest in the Canine Parvovirus once the virus has envolved to a new viral variant that have been detected in several parts of the world $(2,8,10)$. This becomes significant as most of the current available vaccines are manufactured with $\mathrm{CPV}-2$, and the crossimmunity between these different genotypes is not totally understood. Some authors have suggested an update of the virus strains in current vaccines, taking into account the existing partial protection $(2,7,11)$. Because of this, is important the isolation of new Parvovirus circulating variants in order to be used in vaccines manufacture more effective from an immunogenic point of view.

However as Parvovirus viral isolation is difficult, often producing a minimum visible cytopathic effect on cell cultures (6). In fact, in the last stage of infection (8-10 days post infection) it has been observed how specific antibodies in the intestinal lumen frequently kidnap many of the virions, preventing the virus adsorption to cells. Consequently, the isolation of parvovirus from faeces at that stage of infection is often difficult (3).

Comparing the CPE produced by CPV-2c and CPV-2 in CRFK, it was observed that the CPV-2c strain produces less CPE than the CPV-2 strain, furthermore the lowest effect produced by CPV-2c was seen on CRFK cell line. At the view of these results, and taking into account the difficulty in CPV isolation from clinical samples (6), we suggest that, unlike other viruses for CPV isolation, it would be better the use of primary cultures instead of cell lines, as the CPE produced is much clearer in primary canine cell cultures.

Finally, this study describes the first isolation in cellular cultures and characterization of canine parvovirus $2 \mathrm{c}$ genotype, in Latin America. The results pointed here may help to achieve a better understanding of the current status of CPV-2 infection in dogs from Uruguay and Latin America.

\section{REFERENCES}

1. Buonavoglia, C.; Martella, V.; Pratelli, A.; Tempesta, M.; Cavalli, A.; Buonavoglia, D.; Bozzo, G.; Elia, G.; Decaro, N.; Carmichael, L. (2001). Evidence for evolution of canine parvovirus type 2 in Italy. J. Gen. Virol. 82, 3021-3025.

2. Calderon, M.; Mattion, N.; Bucafusco, D.; Fogel, F.; Remorini, P.; La Torre, J. (2009). Molecular characterization of canine parvovirus strains in Argentina: Detection of the pathogenic variant CPV2c in vaccinated dogs. J. Virol. Methods. 159, 141-145.

3. Decaro, N.; Desario, C.; Campolo, M.; Elia, G.; Martella, V.; Ricci, D.; Lorusso, E.; Buonavoglia, C. (2005). Clinical and virological findings in pups naturally infected by canine parvovirus type 2 Glu-426 mutant. $J$ Vet Diagn Invest. 17, 133-138.

4. Decaro, N.; Elia, G.; Campolo, M.; Desario, C.; Lucente, M.; Bellacicco, A.; Buonavoglia, C. (2005). New approaches for the molecular characterization of canine parvovirus type 2 strains. J. Vet. Med. B Infect Dis. Vet. Public Health. 52, 316-319.

5. Hoelzer, K.; Parrish, C. (2010). The emergence of parvoviruses of carnivores. Vet. Res. 41 (39).

6. Mochizuki, M.; Ohshima, T.; Une, Y.; Yachi, A. (2008). Recombination 
7. Between vaccine and Field Strains of Canine Parvovirus is Revealed by Isolation of Virus in Canine and Feline Cell Cultures. J. Vet. Med. Sci. 70 (12), 1305-1314.

8. Ohshima, T.; Mochizuki, M.; Kawakami, K.; Kishi, M.; Tohya, Y.; Mochizuki, M. (2008). Chronological Analysis of Canine Parvovirus Type 2 Isolates in Japan. J. Vet. Med. Sci. 70 (8), 769-775.

9. Pérez, R.; Francia, L.; Romero, V.; Maya, L.; López, I.; Hernández, M. (2007). First detection of canine parvovirus type 2c in South America. Vet. Microbiol. 124, 147-152.
10. Senda, M.; Hirayama, N.; Yamamoto, H. \& Kurata, K. (1986). An improved hemagglutination test for study of canine parvovirus. Vet. Microbiol. 12, 1-6.

11. Streck, A.; Kunzler, C.; Rathje, K.; Zang, L.; Dubina, L.; Wageck, C. (2009). First detection of canine parvovirus type 2c in Brazil. Braz. J. Microbiol. 40(3), 465-469.

12. Truyen, U. (2006). Evolution of canine parvovirus: a need for new vaccines? Vet. Microbiol. 117, 9-13. 